\title{
Stability of the hard-sphere icosahedral quasilattice
}

\author{
H. M. Cataldo \\ Departamento de Física, Facultad de Ciencias Exactas y Naturales, Universidad de Buenos Aires, 1428 Buenos Aires, Argentina \\ C. F. Tejero \\ Facultad de Ciencias Físicas, Universidad Complutense de Madrid, E-28040 Madrid, Spain
}

(Received 31 March 1995)

\begin{abstract}
The stability of the hard-sphere icosahedral quasilattice is analyzed using the differential formulation of the generalized effective liquid approximation. We find that the icosahedral quasilattice is metastable with respect to the hard-sphere crystal structures. Our results agree with recent findings by McCarley and Ashcroft [Phys. Rev. B 49, 15600 (1994)] carried out using the modified weighted density approximation.
\end{abstract}

\section{INTRODUCTION}

The observation in 1984 by Shechtman et al. ${ }^{1}$ of a sharp diffraction pattern in an AlMn alloy with the symmetry of the icosahedron opened a new field in condensed matter physics. Ever since experimental evidence of other materials having sharp diffraction patterns with symmetries forbidden by classical crystallography has continued to grow. The name quasicrystals has been coined to represent systems with perfect order but without periodicity, i.e., quasiperiodic systems.

Since the nonperiodic three-dimensional (3D) Penrose tiling ${ }^{2}$ has a diffraction pattern closely similar to that of icosahedral alloys, it has been extensively studied to account for icosahedral point symmetry and also because of its relative simplicity. The 3D Penrose tiling is usually constructed by projection from a $6 \mathrm{D}$ simple cubic lattice. The projection is performed by first defining an acceptance domain in the $3 \mathrm{D}$ complementary space in order to select what points of the 6D simple cubic lattice are effectively projected to form the $3 \mathrm{D}$ quasilattice. In the present investigation the 3D Penrose tiling has been generated by a special choice of the shape and size of the acceptance domain as described by Elser. ${ }^{2}$ The last step to model a quasicrystal concerns the decoration, i.e., the location of the lattice points forming the quasicrystal and a choice for the pair interaction potential.

Of the many questions about quasicrystals, one concerns the stability of these phases. The first theoretical approaches to such a question ${ }^{3,4}$ were based upon the Landau theory of crystallization, where the free energy is expanded in powers of an order parameter related to density waves with icosahedral symmetry. As a main result it was shown that multicomponent systems are required to achieve stability, a fact that agrees with the experimental findings. ${ }^{3}$

On the other hand and from a more microscopic viewpoint, the analysis of the stability of quasicrystals may be rather difficult on general grounds, but an important simplification occurs if a hard-sphere pair potential is assumed. Indeed, a simple calculation of the maximum packing fraction, i.e., the fraction of the total volume occupied by the spheres, provides an important criterion of the stability chances. For instance, a 3D Penrose tiling with all vertices occupied by identical hard spheres leads to a fluidlike packing fraction and thus it must certainly be discarded as a model of a quasicrystal. Moreover, the interest of considering hard-sphere quasicrystals goes far beyond simplicity. Thus, numerical studies 5 have shown that the crucial criterion for the quasicrystal stability with more realistic, e.g., Lennard-Jones, interactions is the packing fraction of the quasicrystalline hard-sphere decoration.

There are basically two ways of improving the poor packing fraction of the above fully occupied Penrose tiling. These options are to change the decoration ${ }^{6}$ or the acceptance domain. ${ }^{7}$ Both procedures give approximately the same optimal packing fraction $\simeq 0.63$, a value that now indeed justifies a further stability study. Recently, McCarley and Ashcroft ${ }^{8}$ have studied the hard-sphere quasicrystal using a modification of the acceptance domain, ${ }^{7}$ to obtain from the modified weighted density approximation a metastable quasicrystal with respect to the crystalline and fluid phases. Their method is entirely formulated in the $6 \mathrm{D}$ reciprocal space which avoids direct summations over the quasilattice. However, a drawback of this method is that a truncation of the sum in the $6 \mathrm{D}$ reciprocal lattice is needed, the induced estimate error in the free energy per particle of the quasilattice being $\simeq 2 \%$.

In the present paper we will consider the above optimal hard-sphere decoration of the Penrose tiling. Our treatment is based on the generalized effective liquid approximation, ${ }^{9}$ which has been previously applied to perfect hard spheres and hard disk crystals yielding very accurate results as compared to the simulation data. The quasilattice sums are calculated in the $3 \mathrm{D}$ real space using a method which substantially improves convergence errors in comparison to previous $6 \mathrm{D}$ reciprocal lattice treatments.

The paper is organized as follows. In Sec. II we briefly review the generation of the $3 \mathrm{D}$ Penrose tiling which allows us to introduce the hard-sphere decoration. Section III summarizes the generalized effective liquid approximation for the determination of the free energy of the quasicrystal. Our results are presented in Sec. IV together with a discussion concerning the evaluation of quasilattice sums, while in the final Sec. V we gather our conclusions. 


\section{THE ICOSAHEDRAL QUASILATTICE}

In what follows, we consider the icosahedral quasilattice obtained by projecting a subset (to be specified below) of the 6D simple cubic lattice onto a 3D hyperplane ${ }^{2} X_{\|}$. The orientation of $X_{\|}$relative to the lattice is determined by requiring that the projected vectors $\left\{\mathrm{e}_{\|}\right\}$of the $6 \mathrm{D}$ basis vectors $\left\{\mathbf{e}^{j}\right\}(j=1,2, \ldots, 6)$ coincide with the six vertex axes of the icosahedron, i.e.,

$$
\mathbf{e}_{\|}^{j}=P_{\|}^{j k} \mathbf{e}^{k},
$$

where a summation over repeated labels is understood. The matrix representation of the projection operator $P_{\|}$is given by

$$
P_{\|}=\frac{1}{\sqrt{20}}\left(\begin{array}{cccccc}
\sqrt{5} & 1 & 1 & 1 & 1 & 1 \\
1 & \sqrt{5} & 1 & -1 & -1 & 1 \\
1 & 1 & \sqrt{5} & 1 & -1 & -1 \\
1 & -1 & 1 & \sqrt{5} & 1 & -1 \\
1 & -1 & -1 & 1 & \sqrt{5} & 1 \\
1 & 1 & -1 & -1 & 1 & \sqrt{5}
\end{array}\right)
$$

and an elementary calculation leads to $\mid \mathbf{e}^{j} \|^{2}=1 / 2$ $(j=1,2, \ldots, 6)$ and

$$
\begin{gathered}
\cos \left(\mathbf{e}_{\|}^{1}, \mathbf{e}_{\|}^{j}\right)=\sqrt{5} / 5 \\
\cos \left(\mathbf{e}_{\|}^{2+j}, \mathbf{e}_{\|}^{2+k}\right)= \begin{cases}\sqrt{5} / 5, & (j-k= \pm 1, \pm 4) \\
-\sqrt{5} / 5, & (j-k= \pm 2, \pm 3)\end{cases}
\end{gathered}
$$

showing that the vectors $\left\{\mathbf{e}_{\|}^{j}\right\}$ may be identified with the six vertex directions of the icosahedron.

We also consider the 3D hyperplane perpendicular to $X_{\|}, X_{\perp}$, obtained upon projection of the $6 \mathrm{D}$ basis vectors $\left\{\mathbf{e}^{j}\right\}$ by the complementary projector $P_{\perp}$,

$$
\mathbf{e}_{\perp}^{j}=P_{\perp}^{j k} \mathbf{e}^{k}, \quad P_{\perp}^{j k}=\delta^{j k}-P_{\|}^{j k},
$$

where $\delta^{j k}$ is the Kronecker delta. It can be readily shown that the projected vectors $\mathbf{e}_{\perp}^{j}$ may also be identified with the six vertex directions of the icosahedron, but permuted with respect to the projected vectors $\mathbf{e}_{\|}^{j}$, i.e., $\mathbf{e}_{\perp}^{j} \cdot \mathbf{e}_{\perp}^{k}=-\mathbf{e}_{\|}^{j} \cdot \mathbf{e}_{\|}^{k}$ $(j \neq k)$.

Both projections are dense in the $3 \mathrm{D}$ space but a quasilattice of finite density can be constructed by projecting onto $X_{\|}$only those points whose perpendicular space projection lies within a bounded region $\chi$ known as the acceptance domain. $^{2}$ To construct this bounded region we take the 20 distinct triplets $\left\{\mathbf{e}_{\perp}^{i}, \mathbf{e}_{\perp}^{j}, \mathbf{e}_{\perp}^{k}\right\}$ of the projected vectors in $X_{\perp}$. Each triplet defines a rhombohedron of volume $v_{i j k}=\left|\mathbf{e}_{\perp}^{i} \times \mathbf{e}_{\perp}^{j} \cdot \mathbf{e}_{\perp}^{k}\right|$. It is easily found that half of these rhombohedra are "large," i.e., $v_{i j k}=\sqrt{8} \sin (2 \pi / 5) / 10$ and half "small," i.e., $v_{i j k}=\sqrt{8} \sin (4 \pi / 5) / 10$. The disjoint union of these 20 rhombohedra defines a closed convex region $\chi$ named the triacontahedron of volume $v=\sqrt{8}[\sin (2 \pi / 5)$ $+\sin (4 \pi / 5)]$. The triacontahedron is therefore the projection onto $X_{\perp}$ of the unit cell of the $6 \mathrm{D}$ cubic lattice.
The selection of a subset of the $6 \mathrm{D}$ simple cubic lattice is accomplished by requiring that the orthogonal projections $\left\{\mathbf{r}_{\perp}^{j}\right\}$ of the lattice points $\left\{\mathbf{r}^{j}\right\}$ of the $6 \mathrm{D}$ simple cubic lattice lie within the triacontahedron. ${ }^{10}$ This construction yields a quasilattice in $X_{\|}$which may also be regarded as a tiling of the $3 \mathrm{D}$ space by two kinds of rhombohedra. ${ }^{6}$ Using a oneparameter Gaussian approximation for the density peaks of the quasilattice, the one-particle density can be written as

$$
\rho(\mathbf{r})=\left(\frac{\alpha}{\pi}\right)^{3 / 2} \sum_{j} W\left(\mathbf{r}_{\perp}^{j}\right) e^{-\alpha\left(\mathbf{r}-\mathbf{r}_{\|}^{j}\right)^{2}}
$$

where the sum runs over the Bravais lattice vectors of the $6 \mathrm{D}$ simple cubic crystal, $\alpha$ is the inverse width of the Gaussians, and the weight function $W\left(\mathbf{r}_{\perp}^{j}\right)$ is defined by:

$$
W\left(\mathbf{r}_{\perp}^{j}\right)= \begin{cases}1, & \mathbf{r}_{\perp}^{j} \in \chi \\ 0, & \text { otherwise. }\end{cases}
$$

It can be shown that the first three neighbour separations of the icosahedral quasilattice are ${ }^{6} r_{1}^{2}=(3-6 \sqrt{5} / 5) a^{2}$, $r_{2}=a$, and $r_{3}^{2}=(2-2 \sqrt{5} / 5) a^{2}$, where $a=\sqrt{2} / 2$. Their average coordination numbers are $2 / \tau^{2}, 6$, and 6 , respectively, with $\tau$ denoting the golden ratio $\tau=(1+\sqrt{5}) / 2$. By locating a hard sphere of diameter $\sigma=r_{1}$ at every vertex of the quasilattice, the packing fraction (the fraction of the total volume occupied by the spheres) is $\simeq 0.14$, i.e., a packing fraction characteristic of a fluid phase. On the other hand, if we look for accommodating hard spheres of diameter $\sigma=a$ at every vertex, the short distance $r_{1}$ does not allow this. But since the frequency of the nearest-neighbor separation is small, a better packing of hard spheres in the quasilattice can be obtained if one of the two vertices of each $r_{1}$ bond is left vacant. These short bonds form closed rings of 10 links and chains of even or odd links. If we also require that two adjacent sites cannot be empty there are only two ways of placing the hard spheres on rings and chains. In the present investigation we have randomly located a hard sphere on a vertex of every ring and in an end point of each chain. This determines the accommodation of the remaining hard spheres, the effect of taking different initial localizations having a negligible effect on our results as the number of vertices of the quasilattice increases. It can be shown ${ }^{6}$ that with this procedure, the volume occupied by the hard spheres increases leading to a hard-sphere packing fraction $\eta \simeq 0.629$, which is close to the random packing fraction ( $\simeq 0.64$ ) and below the packing fraction of the crystal structures $(\simeq 0.74$ and $\simeq 0.68$ for the fcc and bcc crystals, respectively). This is, to our knowledge, the best icosahedral packing fraction of identical hard spheres reported so far.

\section{FREE ENERGY OF THE HARD-SPHERE ICOSAHEDRAL QUASILATTICE}

In recent years, the freezing of hard spheres into perfect crystals has been successfully described by several nonperturbative density functional theories. We here consider the stability of the hard-sphere icosahedral quasilattice described in Sec. II within one of such approaches, the generalized effective liquid approximation, which is now briefly summarized. 
The Helmholtz free energy $F$ of a solid characterized by a one-particle density $\rho(\mathbf{r})$ is a functional of $\rho(\mathbf{r})$, denoted by $F=F[\rho]$, which can be split as $F[\rho]=F_{\text {id }}[\rho]+F_{\text {ex }}[\rho]$, where

$$
\beta F_{\mathrm{id}}[\rho]=\int d \mathbf{r} \rho(\mathbf{r})\left[\ln \left\{\Lambda^{3} \rho(\mathbf{r})\right\}-1\right]
$$

is the ideal contribution with $\beta=1 / k_{B} T$ the inverse temperature and $\Lambda$ the thermal de Broglie wavelength and

$$
\begin{aligned}
\beta F_{\mathrm{ex}}[\rho]= & -\int d \mathbf{r} \rho(\mathbf{r}) \int d \mathbf{r}^{\prime} \rho\left(\mathbf{r}^{\prime}\right) \\
& \times \int_{0}^{1} d \lambda(1-\lambda) c\left(\mathbf{r}, \mathbf{r}^{\prime} ;[\lambda \rho]\right)
\end{aligned}
$$

is the excess term. In Eq. (3.2) $c\left(\mathbf{r}, \mathbf{r}^{\prime} ;[\lambda \rho]\right)$ is the direct correlation function of the solid and $\lambda(0 \leqslant \lambda \leqslant 1)$ is a parameter defining a linear path of integration in the space of density functions $\rho_{\lambda}(\mathbf{r})=\lambda \rho(\mathbf{r})$ connecting a zero reference density to the one-particle density $\rho(\mathbf{r})$ of the solid. The equilibrium solid density $\rho(\mathbf{r})$, determined by functional differentiation, is the minimimum value of $F[\rho]$ at constant average density. This variational calculation implies the direct correlation function of the solid which is the only unknown in (3.1) and (3.2) and hence some explicit approximations for $F_{\text {ex }}[\rho]$ are required.

Based on the similarity of the thermodynamic properties of the solid and fluid phases, the generalized effective liquid approximation first maps the excess free energy per particle of the solid onto that of some effective liquid, i.e.,

$$
\begin{aligned}
\frac{1}{N} \int d \mathbf{r} \rho(\mathbf{r}) & \int d \mathbf{r}^{\prime} \rho\left(\mathbf{r}^{\prime}\right) \int_{0}^{1} d \lambda(1-\lambda) c\left(\mathbf{r}, \mathbf{r}^{\prime} ;[\lambda \rho]\right) \\
= & \hat{\rho} \int d \mathbf{r} \int_{0}^{1} d \lambda(1-\lambda) c(|\mathbf{r}| ; \lambda \hat{\rho}),
\end{aligned}
$$

where $\hat{\rho}$ is the density of the effective liquid, $N=\int d \mathbf{r} \rho(\mathbf{r})$ is the number of particles, and $c(|\mathbf{r}| ; \lambda \hat{\rho})$ is the direct correlation function of the liquid. Equation (3.3) is referred to as the thermodynamic mapping.

In a second step, the generalized effective liquid approximation defines a structural mapping in which the direct correlation function of the solid is mapped onto that of a liquid. However, this mapping cannot be done directly because the direct correlation function of the liquid is translationally invariant while that of the solid is not. But taking into account that in (3.2) the direct correlation function of the solid appears doubly weighted by the solid density, the difficulty is overcome by defining the structural mapping as

$$
\begin{aligned}
& \int d \mathbf{r} \rho(\mathbf{r}) \int d \mathbf{r}^{\prime} \rho\left(\mathbf{r}^{\prime}\right) c\left(\mathbf{r}, \mathbf{r}^{\prime} ;[\rho]\right) \\
& =\int d \mathbf{r} \rho(\mathbf{r}) \int d \mathbf{r}^{\prime} \rho\left(\mathbf{r}^{\prime}\right) c\left(\left|\mathbf{r}-\mathbf{r}^{\prime}\right| ; \hat{\rho}[\rho]\right) .
\end{aligned}
$$

With (3.2)-(3.4) a self-consistent nonlinear integral equation is obtained for the determination of $\hat{\rho}[\rho]$ in terms of $\rho(\mathbf{r})$ and the direct correlation function of the liquid. The complicated functional dependence $\hat{\rho}[\rho]$ can be simplified if $\rho(\mathbf{r})$ is described in terms of a single order parameter $\alpha$ as in (2.4) in which case $\hat{\rho}$ becomes an ordinary function of $\alpha$. The equilibrium solid density (i.e., $\alpha$ ) is then determined by minimizing at constant average density the solid free energy with respect to the Gaussian width parameter $\alpha$ for a given crystal structure.

As explained elsewhere, ${ }^{11}$ the nonlinear integral equation for the determination of $\hat{\rho}[\rho]$ can be further transformed into a system of two coupled nonlinear differential equations in $\hat{\eta}(\lambda)$

$$
\hat{\eta}^{\prime}(\lambda)=\frac{z(\lambda)-\psi(\hat{\eta}(\lambda))}{\lambda \psi^{\prime}(\hat{\eta}(\lambda))}
$$

and $z(\lambda)$

$$
z^{\prime}(\lambda)=\Phi(\hat{\eta}(\lambda))
$$

where $\hat{\eta}(\lambda)=\pi \hat{\rho}(\lambda) \sigma^{3} / 6$ is the effective liquid packing fraction and $\sigma$ is the hard-sphere diameter.

Using the one-parameter approximation (2.4) for the oneparticle density of the quasilattice, $\Phi(\hat{\eta}(\lambda))$ is given by

$$
\begin{aligned}
\Phi(\hat{\eta}(\lambda))= & -\frac{1}{N} \sum_{i=1}^{N} \sum_{j=1}^{N} W\left(\mathbf{r}_{\perp}^{i}\right) W\left(\mathbf{r}_{\perp}^{j}\right) \\
& \times \int_{0}^{\infty} d R R c(R ; \hat{\eta}(\lambda)) S\left(R ; \alpha, r_{i j}\right),
\end{aligned}
$$

where $r_{i j}=\left|\mathbf{r}_{\|}^{i}-\mathbf{r}_{\|}^{j}\right|$ and

$$
\begin{aligned}
S\left(R ; \alpha, r_{i j}\right)= & {\left[\frac{\alpha}{2 \pi r_{i j}^{2}}\right]^{1 / 2}\left\{\exp \left[-\alpha\left(R-r_{i j}\right)^{2} / 2\right]\right.} \\
& \left.-\exp \left[-\alpha\left(R+r_{i j}\right)^{2} / 2\right]\right\}
\end{aligned}
$$

In (3.5) and (3.6) the prime denotes the derivative with respect to the argument and $\psi(\hat{\rho}) / \beta$ is the excess free energy per particle of the fluid phase. For the latter we will use the Carnahan-Starling compressibility factor to obtain $\psi$ by thermodynamic integration of the equation of state while the Percus-Yevick equation is used for the structure of the fluid phase, i.e., the direct correlation function. Equations (3.5) and (3.6) have to be integrated numerically from $\lambda=0$ to $\lambda=1$ with initial conditions $\hat{\eta}(0)=z(0)=0$ and the excess free energy per particle of the quasilattice (3.2) is finally determined as $\psi(\hat{\eta}(1))$.

\section{RESULTS}

Before looking for a numerical solution of (3.5) and (3.6), we deal with a delicate point concerning the convergence of the quasilattice sums in (3.7). In order to emphasize it let us rewrite the right-hand side of Eq. (3.7) in the form

$$
\frac{1}{N} \sum_{i=1}^{N} \sum_{j=1}^{N} A\left(r_{i j}\right)
$$

We first note that for a Bravais lattice (4.1) reduces to 


$$
\sum_{j} z_{j} A\left(r_{j}\right)
$$

where the sum runs over spherical shells of sites centered around the site at the origin, $r_{j}$ is the distance of shell $j$ to the origin, and $z_{j}$ is the number of sites at the $j$ th shell. In passing from (4.1) to (4.2) the translational symmetry of the Bravais lattice has been used. Moreover, since $A\left(r_{j}\right)$ decreases rapidly with distance, only a relatively small number of shells around the origin give a non-negligible contribution to the sum leading to a rapid convergence of (4.2).

But if the lattice does not have translational symmetry, the evaluation of (4.1) becomes a delicate numerical problem if one looks for achieving a rapid convergence. For instance, if we consider a quasilattice of $N$ sites the convergence of (4.1) [resulting from considering the $N(N-1) / 2$ different pairs $i$ $\neq j]$ becomes so slow as $N$ increases that it remains unreachable through usual computational efforts. This is also the case for a Bravais lattice as it may be tested by evaluating the sum (4.1) which, on the other hand, can be easily determined through (4.2).

As stated above, a possible way for dealing with the quasilattice $\operatorname{sum}^{8}$ is to use the $6 \mathrm{D}$ reciprocal lattice. However, this procedure only provides a partial solution to the convergence of the sum since it is necessary to truncate the sum at some maximum value of the reciprocal lattice vector leading to an estimate error in the free energies of about $2 \%$.

We here propose an alternative solution to the convergence problem of (4.1) which provides a substantial reduction of errors and computation time in the determination of the quasicrystal free energy. By starting with the 6D simple cubic lattice, we construct the quasilattice using the projection formalism described in Sec. II. Let $M \gg 1$ be the number of the lattice points generated in the quasilattice and draw a spherical surface containing almost all of the lattice points. Inside the sphere we construct a concentric sphere with $N \gg 1$ $(N<M)$ lattice points.

By rewriting (4.1) as

$$
A(0)+\frac{1}{N} \sum_{i=1}^{N}\left[\sum_{j \neq i} A\left(r_{i j}\right)\right],
$$

where we have separated the $r_{i j}=0$ contributions, we calculate the $i \neq j$ terms by first choosing a lattice point $i$ $(i=1,2, \ldots, N)$ inside the sphere and then summing consecutively over all neighboring $j$ lattice points until the relative error of the sum in brackets in (4.3) is less than a prefixed value. For lattice points $i$ well inside the sphere, our procedure takes into account all the relevant "interactions" in (4.1). However, if the lattice point $i$ lies near the surface of the sphere, our procedure overestimates the "interactions" in (4.1) because the sum in brackets contains points outside the sphere. We have found that these boundary errors can be reduced by increasing $N$ (and therefore $M$ ), in such a way that for $M=22000$ and $N=10000$, the estimate error of the free energy per particle of the quasilattice is about $0.1 \%$. Under such conditions, the computation time needed for evaluating the variational free energy (for each pair $\alpha-\eta$ ) is around $20^{\prime}$ CPU in a VAX 9000.

In Fig. 1, the variational free energy per particle of the quasicrystal $\beta \phi=\beta F / N-3 \ln (\Lambda / \sigma)+1$ is represented versus

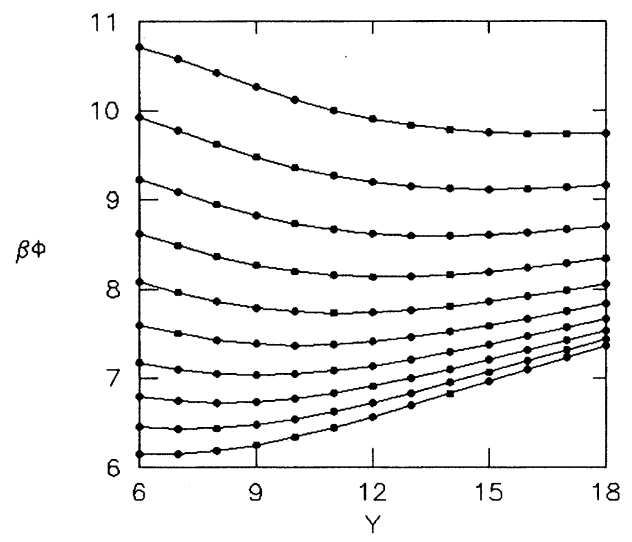

FIG. 1. Variational free energy per particle of the quasilattice $\beta \phi=\beta F / N-3 \ln (\Lambda / \sigma)+1$ vs the Gaussian width parameter $Y=\left(\alpha \sigma^{2} / 2\right)^{1 / 2}$ for packing fractions $\eta=0.51-0.60$, by steps of 0.01 (from bottom to top).

$Y=\left(\alpha \sigma^{2} / 2\right)^{1 / 2}$ for different packing fractions. We have found minima for the quasicrystal free energy as a function of the Gaussian width parameter $\alpha$ for $\eta \geqslant 0.51$, i.e., a stable or metastable quasicrystalline phase. Similarly to Ref. 8 we have found that the quasicrystal turns out to be the more localized phase since the minima are always situated at greater $Y$ values than the compact fcc crystalline phase. Our results are gathered in Table I where the free energy and the Gaussian width parameter at the free energy mimimum of the quasicrystal are compared to those of the fcc crystal.

In Fig. 2 we represent the solid free energy per particle versus the packing fraction $\eta$ for the three solid phases (fcc, bcc, and quasicrystal). We also include in the figure the fluid free energy obtained by thermodynamic integration of the Carnahan-Starling equation of state. It is seen that the quasicrystal is always metastable with respect to the remaining phases, the gaps of the free energy being somewhat less than those reported by $\mathrm{M}^{\mathrm{c}} \mathrm{Carley}$ and Ashcroft. ${ }^{8}$

\section{CONCLUSIONS}

We have analyzed the stability of a hard-sphere quasicrystal obtained from a simple decoration of the 3D Penrose

TABLE I. Free energy $\beta \phi=\beta F / N-3 \ln (\Lambda / \sigma)+1$ and Gaussian width parameter $Y=\left(\alpha \sigma^{2} / 2\right)^{1 / 2}$ at the free energy minimum for the quasicrystal $(q)$ and the fcc crystal $(c)$ phases at different packing fractions $\eta$.

\begin{tabular}{lcccc}
\hline \hline$\eta$ & $\beta \phi_{q}$ & $Y_{q}$ & $\beta \phi_{c}$ & $Y_{c}$ \\
\hline 0.51 & 6.15 & 6.5 & 5.28 & 5.9 \\
0.52 & 6.43 & 7.0 & 5.48 & 6.5 \\
0.53 & 6.73 & 8.0 & 5.68 & 7.0 \\
0.54 & 7.04 & 9.0 & 5.88 & 7.6 \\
0.55 & 7.37 & 10.0 & 6.09 & 8.2 \\
0.56 & 7.74 & 11.0 & 6.31 & 8.9 \\
0.57 & 8.14 & 12.0 & 6.53 & 9.7 \\
0.58 & 8.59 & 13.5 & 6.76 & 10.6 \\
0.59 & 9.12 & 15.0 & 7.01 & 11.6 \\
0.60 & 9.74 & 16.5 & 7.26 & 12.6 \\
\hline \hline
\end{tabular}




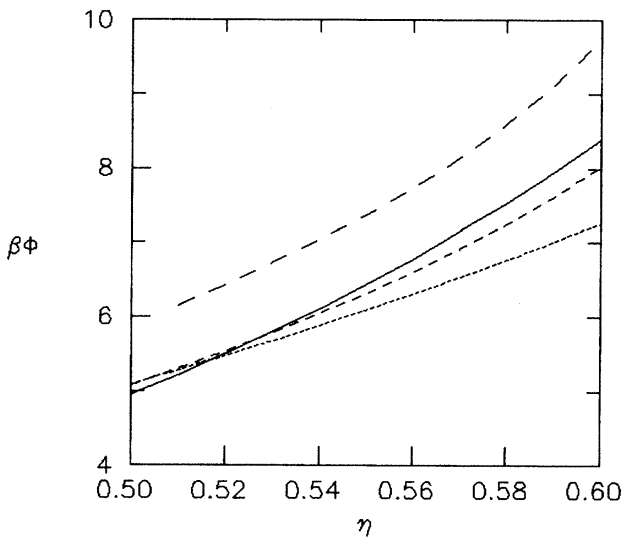

FIG. 2. Free energy per particle $\beta \phi=\beta F / N-3 \ln (\Lambda / \sigma)+1$ vs the packing fraction $\eta$ for the solid phases: fcc (dotted line), bcc (medium dashed line), and quasicrystal (dashed line), and for the fluid phase (continuous line).

tiling which has been designed for optimizing the packing fraction. The stability has been analyzed using the generalized effective liquid approximation. A simple method for evaluating the quasilattice sums in the $3 \mathrm{D}$ real space has been formulated. The method minimizes the boundary effects of finite quasilattices leading to a substantially better convergence than previous works.

Our results show that the quasicrystal is metastable with respect to the crystalline and fluid phases. Such results agree with recent reported ${ }^{8}$ calculations for a hard-sphere quasicrystal obtained from the modified weighted density approximation. Therefore, within these nonperturbative density functional theories entropy is insufficient to stabilize onecomponent quasicrystals.

Since all known quasicrystals have complex metallic alloy phase structures it has been argued that for the stability of quasicrystals it is necessary to have at least two classes of atoms. The generalization of the one-component quasicrystal structure to an ordered two-component structure has been investigated by $\mathrm{M}^{\mathrm{C}}$ Carley and Ashcroft, ${ }^{8}$ who concluded that small changes in the diameter ratio of the two-component hard-sphere quasicrystal are not a stabilizing factor. It should be expected that energetic contributions resulting from considering more realistic interactions would propitiate stability. This possibility seems to be ruled out using the well-known perturbation schemes when applied to quasicrystals in view of the great free energy differences between the crystalline and quasicrystalline hard-sphere phases. Thus, the stability of quasicrystal structures within the modern density functional approaches is at present an open question.

\section{ACKNOWLEDGMENTS}

We thank R. Brito and J. M. R. Parrondo for useful discussions. H. M. Cataldo has been supported by Grants Nos. PID 97/93 from Consejo Nacional de Investigaciones Científicas y Técnicas (Argentina) and EX100 from Universidad de Buenos Aires. C. F. Tejero acknowledges the DGICYT, Spain (Grant No. PB91-0378) for its financial support.
${ }^{1}$ D. Shechtman, I. Blech, D. Gratias and J. W. Cahn, Phys. Rev. Lett. 53, 1951 (1984).

${ }^{2}$ V. Elser, Acta Crystallog. A 42, 36 (1986).

${ }^{3}$ N. D. Mermin and S. M. Troian, Phys. Rev. Lett. 54, 1524 (1985).

${ }^{4}$ P. A. Kalugin, A. Y. Kitaev, and L. S. Levitov, J. Phys. (Paris) Lett. 46, L601 (1985); Pis'ma Zh. Éksp. Teor. Fiz. 41, 119 (1985) [JETP Lett. 41, 145 (1985)].

${ }^{5}$ J. Roth, R. Schilling, and H. R. Trebin, Phys. Rev. B 41, 2735 (1990).
${ }^{6}$ C. L. Henley, Phys. Rev. B 34, 797 (1986).

${ }^{7}$ C. Oguey and M. Duneau, Europhys. Lett. 7, 49 (1988).

${ }^{8}$ J. S. M Marley and N. W. Ashcroft, Phys. Rev. B 49, 15600 (1994).

${ }^{9}$ J. F. Lutsko and M. Baus, Phys. Rev. A 41, 6647 (1990).

${ }^{10}$ The triacontahedron has to be arbitrarily displaced from its original position in order to avoid spurious symmetries in the quasilattice. A two-dimensional analog of this situation is discussed by M. V. Jarić, Phys. Rev. B 34, 4685 (1986).

${ }^{11}$ C. F. Tejero and J. A. Cuesta, Phys. Rev. E 47, 490 (1993). 